\title{
Nis-Nim Intercomparison of Tungsten Strip Lamps
}

\author{
K. Ali*, Y.Zundong, B.Xing and J.Wu \\ *National Institute for Standards (NIS-Egypt) \\ National Institute for Metrology (NIM-China)
}

One of the principal responsibilities of the National Institute for Standards is to maintain, establish and develop the national temperature standards and using them for calibration purposes. To meet this responsibility in the high temperature range, above the freezing point of silver, the National Institute for Standards (NIS-Egypt), and the National Institute for Metrology (NIM-China), have undertaken an intercomparison of tungsten strip lamps for the radiance temperature scale maintained at the two laboratories. This intercomparison was carried out at NIM in the temperature rang from 800 up to $2200^{\circ} \mathrm{C}$, using two calibrated lamps from NIS (one evacuated and the other is gas filled). It was found that the difference ranged from $0.34{ }^{\circ} \mathrm{C}$ at $800{ }^{\circ} \mathrm{C}$ to $5.30{ }^{\circ} \mathrm{C}$ at $2200^{\circ} \mathrm{C}$.

\section{Introduction:}

Above the freezing point of silver $\left(961.78{ }^{\circ} \mathrm{C}\right)$, the International Temperature Scale of 1990 (ITS-90) is realized using a radiation thermometer and Planck's radiation law in a ratio form. The temperature of a source is derived from the ratio of the spectral radiance of the source to the spectral radiance of a fixed-point blackbody source at one of the defining fixed points silver, gold or copper [1]. Thus:

$$
\frac{L_{90}(x)}{L_{t}}=\frac{\exp \left(c_{2} / \lambda T_{t}\right)-1}{\exp \left[c_{2} / \lambda T_{90}(x)\right]-1}
$$

Where $T(x)$ is the radiance temperature of the fixed point blackbody source in Kelvin

$\mathrm{T}_{t}$ is the radiance temperature of the test source in Kelvin

$\mathrm{L}_{90}(\mathrm{x})$ is the radiance intensity from the fixed point blackbody

$\mathrm{L}_{\mathrm{t}}$ is the radiance intensity from the test source

$\lambda$ is the wavelength at which the comparison has been made

$\mathrm{c}_{2}$ is the second radiation constant $0.014388 \mathrm{~m} . \mathrm{K}$

$(\mathrm{x})$ is the silver, gold or copper point 
The radiance temperature of a source is defined as the temperature of a blackbody (emissivity of 1) having the same radiance at the stated wavelength.

As a result of the normal variations in properties of the components used to construct the radiation thermometer, particularly in the sensitivity of the photodetector, it is necessary to calibrate a new instrument in order to achieve moderate levels of accuracy. A further check is that; a recalibration should be repeated at regular intervals throughout its working life to maintain its performance, as very few pyrometers posses adequate long term stability. The establishment of a history of calibration results for particular pyrometer also causes sudden changes due to vibration or mechanical shocks. It is usual to calibrate an instrument against a standard source, that is, a device at a known temperature or producing a known amount of thermal radiation. As a practical standard, however, a blackbody source has a number of disadvantages. Together with the necessary power supplies and instrumentation, it forms a large and rather cumbersome system to be transported from one calibration laboratory to another. When the temperature is changed, it may take several hours to reach equilibrium. At higher temperatures, above $1000{ }^{\circ} \mathrm{C}$, the contact thermometers become increasingly less stable and therefore unsuitable for this purpose.

As an alternative, tungsten ribbon lamps (also known as tungsten strip lamps) are commonly used as standard radiation sources. A lamp consists essentially of an electrically heated flat tungsten filament in a transparent glass envelope.The envelope may be evacuated to prevent oxidation or contamination of the filament, in which case the operating range of the lamp extends from about $700^{\circ} \mathrm{C}$ to about $1700^{\circ} \mathrm{C}$, determined by the rate of evaporation of tungsten from the filament. This suppressed by filling the lamp with an inert gas, normally pure argon, whereby stable operation at temperature up to $2300{ }^{\circ} \mathrm{C}$ is achieved. Despite the increasing use of blackbody sources as radiance temperature standards, lamps are still widely used. This is due to their stability and their small size, making them suitable for both maintaining the scale and as transfer standards, especially for the international intercomparison [2,3].

High-grade tungsten-ribbon lamps were calibrated against the freezing points of silver, gold and copper and then used as a transfer standard for calibrating the tungsten-ribbon lamps, blackbody sources and visual pyrometers. During 1999 to 2000 NIS and NIM undertook such an intercomparison, designed to check the difference between the two laboratories. 


\section{Experimental:}

NIS lamps were high stability types, with a $1.5 \mathrm{~mm}$ filament width and $80 \mathrm{~mm}$ diameter window. One of them No. PC907 was evacuated and the other No. PC908 was gas filled [4]. The alignment indicator was in the form of a notch in the filament and a white spot on the rear window as shown in Fig.(1). They were set up with the positive side of the voltage supply connected to the upper end of the lamp filament.

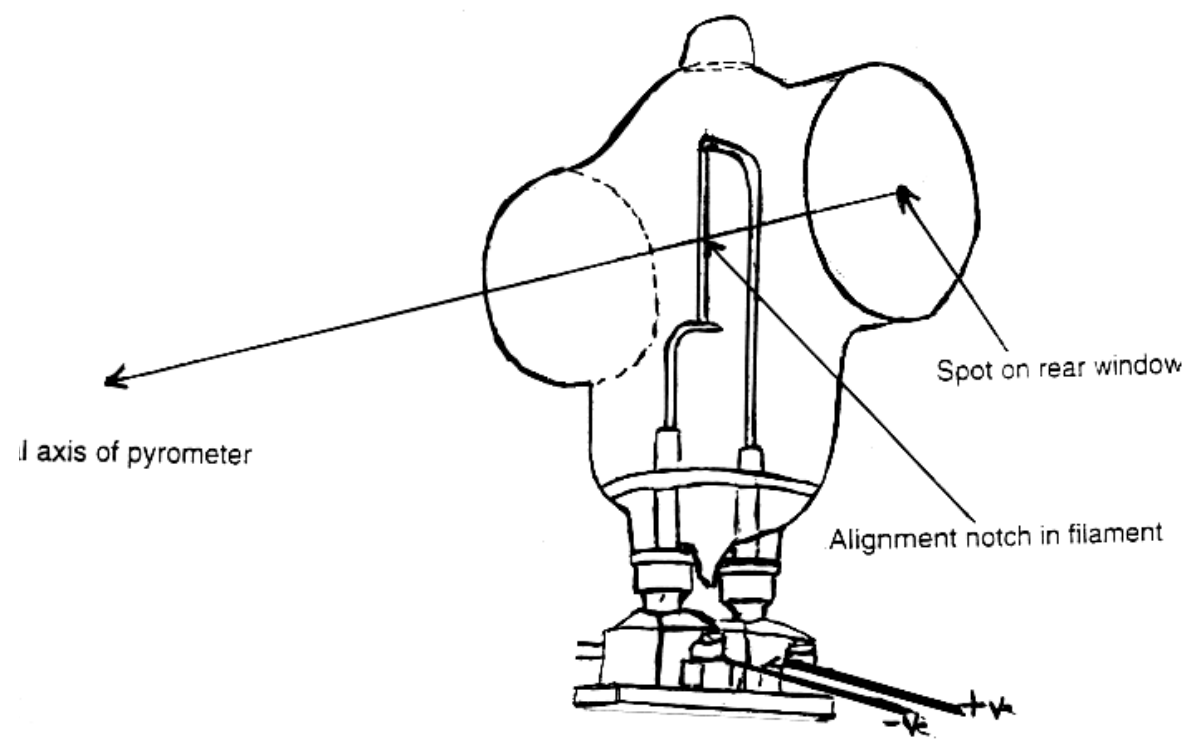

Fig. (1): NIS lamp.

NIM Lamps were high stability types with a $2.0 \mathrm{~mm}$ filament width and $80 \mathrm{~mm}$ diameter window. The first lamp No. C710 was evacuated and the second one No. C905 was gas filled. The alignment indicators were in the form of a notch in the filament and a white spot on the rear window. Fig.(2) shows a schematic for the lamp and the field of view during the measurements.

A DC Photoelectric comparator pyrometer No. RT9031-5 was used for comparing the lamps. It has a $70 \mathrm{~mm}$ diameter objective lens of $140 \mathrm{~mm}$ focus and about $0.75 \mathrm{~mm}$ target diameter when objective distance is $40 \mathrm{~mm}$.

\section{Theory and Discussion}

Annealing of NIS lamps was done after transportation and six runs for comparison of the radiance temperature were carried out for each pair of the lamps using a DC photoelectric comparator. 

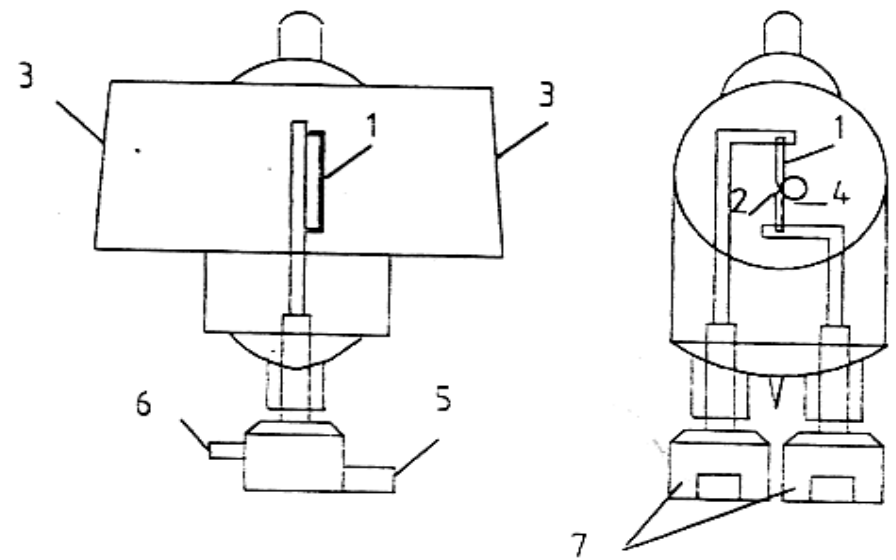

Fig.(2): NIM lamp with 1: tungsten, 2:small notch, 3: window, 4:spot on rear window, 5: terminal, 6: cooled-water pipe, 7:water cooled base.

Each lamp was put on an optical mount (which allows full $x-y-z$ and rotational adjustments and screw into place). The power leads from the output terminal of the power supply with the positive one were connected to the positive lamp terminal and the negative one to the negative lamp terminal. A calibrated $0.01 \Omega$ standard resistor was incorporated into the circuit so that the current through the filament can be accurately measured for the evacuated lamps and another $0.001 \Omega$ for the gas filled lamps. The base of the lamps was connected to a water cooling system to control the temperature of the base at 20 $\pm 0.1{ }^{\circ} \mathrm{C}$.

The alignment and focus of each lamp filament in the field of view of the comparator was adjusted. The angle of the alignment must be adjusted such that the spot on the rear window is directly behind the filament when viewing along the optical axis of the comparator as shown in Fig.(3). The alignment and focus were reviewed at each series of measurements and each temperature.

Measurement were carried out using NIM radiation thermometer of $660.95 \mathrm{~nm}$ wavelength, which corrected to NIS thermometer of $662.4 \mathrm{~nm}$ wavelength according to the original calibration of NIS lamps. This was performed by using the calculation of the radiance temperature correction when viewing a lamp with pyrometers operating at different wavelengths. If the radiance temperature of the lamp at wavelength $\lambda_{0}$ is $T_{0}$ then the corresponding temperature $T_{\lambda}$ at wavelength $\lambda$ is given by the equation:

$$
1 / T_{o}+\lambda_{o} \ln \left[\tau \cdot \varepsilon\left(\lambda_{o}\right)\right] / c_{2}=1 / T_{\lambda}+\lambda \ln [\tau . \varepsilon(\lambda)] / c_{2}
$$


Where $\tau$ is the transmission of the optical window of the lamp (generally assumed to be 0.91$)$ and $\varepsilon\left(\lambda_{0}\right)$ and $\varepsilon(\lambda)$ are the emissivities of the tungsten filament at wavelengths $\lambda_{o}$ and $\lambda$ respectively $[5,6]$.

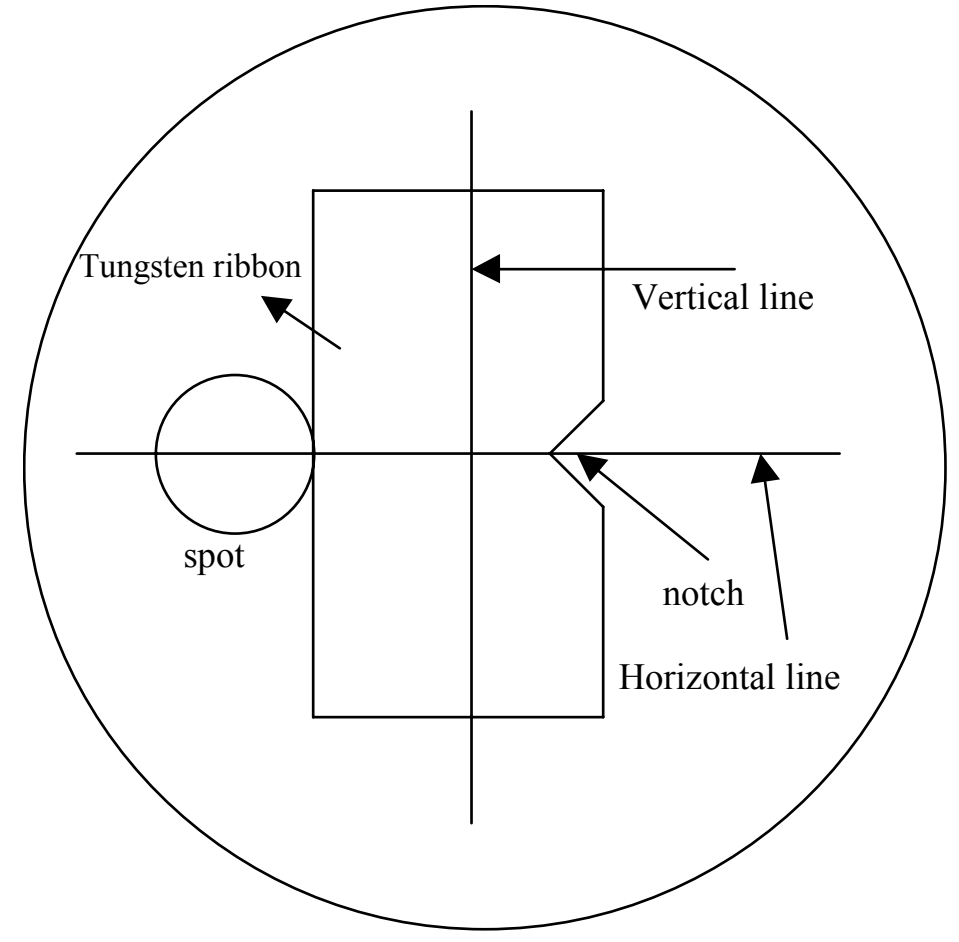

Fig.(3): Field of view of the comparator.

Expanding the last term in equation (2) and assuming that the transmittance of the envelope is independent of wavelength, then;

$$
\ln [\tau . \varepsilon(\lambda)]=\ln \left[\tau \cdot \varepsilon\left(\lambda_{o}\right)\right]+\ln \left[\varepsilon(\lambda) / \varepsilon\left(\lambda_{o}\right)\right]
$$

Gives after rearrangement

$$
c_{2}\left[\frac{1}{T_{o}}-\frac{1}{T_{\lambda}}\right]=\left(\lambda-\lambda_{o}\right) \cdot \ln \left[\tau \cdot \varepsilon\left(\lambda_{o}\right)\right]+\lambda \cdot \ln \frac{\varepsilon(\lambda)}{\varepsilon\left(\lambda_{o}\right)}
$$

This can be used to calculate the correction in the general case. For small differences in the wavelengths, it is convenient to write this in terms of a correction factor $\mathrm{dT}_{\lambda} / \mathrm{d} \lambda$. Expanding $\varepsilon(\lambda)$ as a Taylor series about $\lambda_{\mathrm{o}}$ 


$$
\varepsilon(\lambda)=\varepsilon\left(\lambda_{\mathrm{o}}\right)+\left(\lambda-\lambda_{\mathrm{o}}\right) \cdot \mathrm{d} \varepsilon(\lambda) / \mathrm{d} \lambda+\ldots \ldots
$$

the last term in equation (3) becomes;

$$
\lambda \cdot \ln \frac{\varepsilon(\lambda)}{\varepsilon\left(\lambda_{o}\right)}=\lambda \cdot \ln \left[1+\left(\lambda-\lambda_{o}\right) \cdot \frac{d \varepsilon(\lambda)}{d \lambda} / \varepsilon\left(\lambda_{o}\right)+\ldots \ldots . .\right]
$$

For small wavelength differences, the logarithmic term may be expanded and higher order terms may be neglected, giving

$$
\begin{aligned}
\lambda \cdot \ln \frac{\varepsilon(\lambda)}{\varepsilon\left(\lambda_{o}\right)} & =\lambda \cdot \ln \left(\lambda-\lambda_{o}\right) \cdot \frac{d \varepsilon(\lambda)}{d \lambda} / \varepsilon\left(\lambda_{o}\right) \\
& =\left(\lambda-\lambda_{o}\right) \cdot \frac{d \varepsilon(\lambda)}{d \ln \lambda} / \varepsilon\left(\lambda_{o}\right)
\end{aligned}
$$

Substituting this expression into equation (3)

$$
\begin{gathered}
c_{2}\left[\frac{1}{T_{o}}-\frac{1}{T_{\lambda}}\right]= \\
\frac{d T_{\lambda}}{d \lambda}=\lim _{\lambda \rightarrow \lambda_{o}}\left[\frac{\left.T_{\lambda}-T_{o}\right) \cdot \ln \left[\tau \cdot \varepsilon\left(\lambda_{o}\right)\right]+\frac{d \varepsilon(\lambda)}{d \varepsilon\left(\lambda_{o}\right)} / \varepsilon\left(\lambda_{o}\right)}{\lambda-\lambda_{o}}\right]
\end{gathered}
$$

The correction factor is given by the limit as $\lambda$ approaches $\lambda_{0}$, thus;

$$
=\frac{T_{\lambda}^{2}}{c_{2}} \cdot\left(\ln [\tau . \varepsilon(\lambda)]+\frac{d \ln \varepsilon(\lambda)}{d \ln (\lambda)}\right)
$$

Equation (4) is the final expression for the correction used to correct the wavelength. Table.1.and Fig.(4).show the results obtained from the comparison of the two laboratories. 

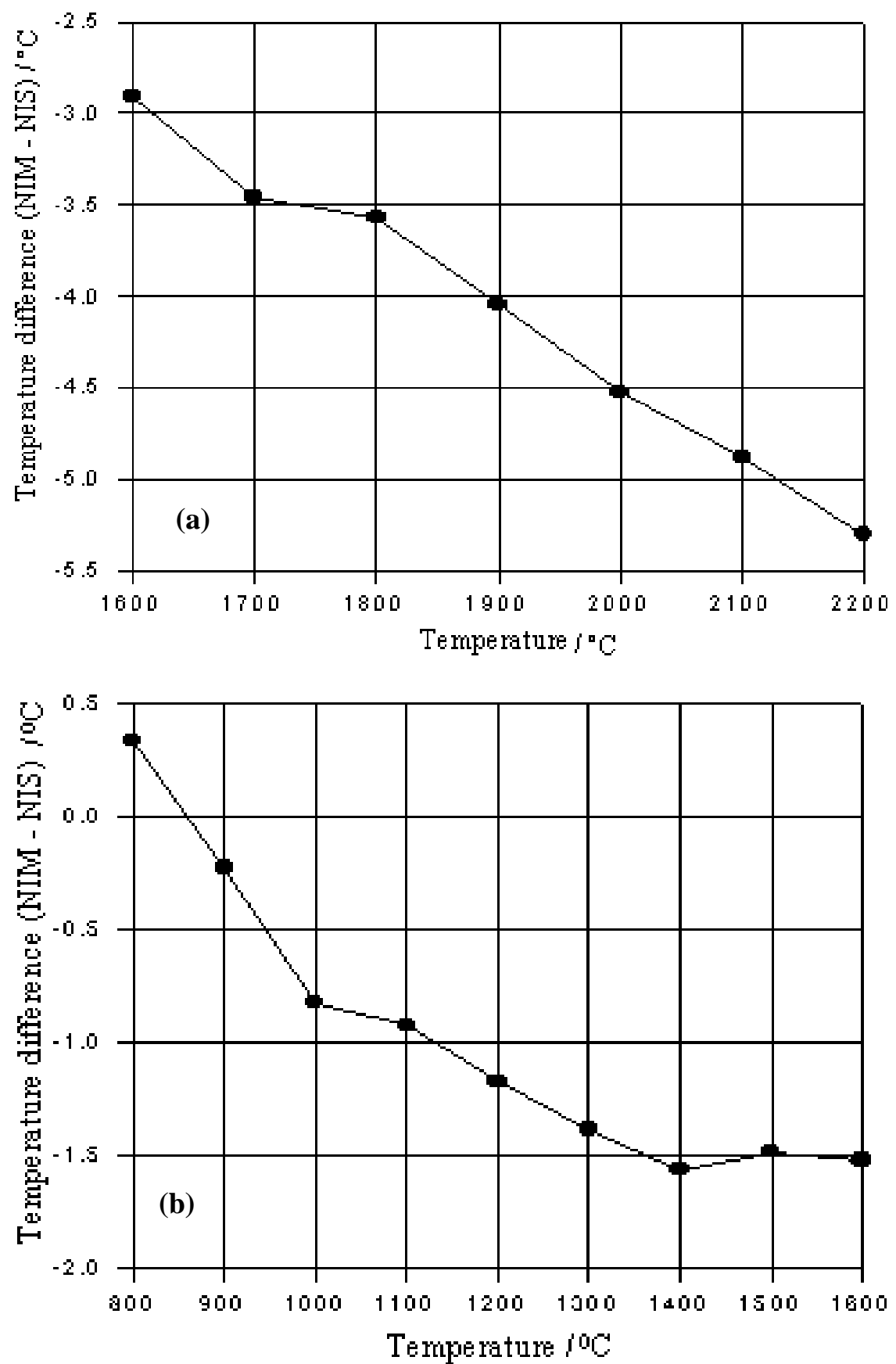

Fig.(4): Difference between NIM-NIS for the evacuated lamp (a) and gas filled (b) 


\section{Conclusion:}

The results of the intercomparison show the differences in the radiance temperature of tungsten strip lamps in the temperature range from 800 up to $2200{ }^{\circ} \mathrm{C}$. The radiance temperature scale maintained on the two laboratories agreed to less than $0.1 \%$ of temperature, although NIS scale was higher than NIM scale. This agreement gives a confidence in the principle and execution of the ITS-90 realizations over this temperature range.

Table.(1) Obtained results for NIS and NIM intercomparison

\begin{tabular}{|c|c|c|c|c|}
\hline \multicolumn{5}{|c|}{ Vacuum lamps } \\
\hline $\mathrm{t}^{\circ} \mathrm{C}$ & \multicolumn{2}{|c|}{ NIM } & NIS & $\Delta \mathrm{t}$ (NIM-NIS) ${ }^{\circ} \mathrm{C}$ \\
\hline & $\mathrm{I}$ A(660.95 nm) & $\mathrm{I}$ A(662.4 nm) & $\mathrm{I}$ A(662.4 nm) & \\
\hline 800 & 3.8581 & 3.8587 & 3.8570 & 0.34 \\
900 & 4.4287 & 4.4296 & 4.4310 & -0.22 \\
1000 & 5.1434 & 5.1446 & 5.1510 & -0.82 \\
1100 & 5.9930 & 5.9947 & 6.0030 & -0.92 \\
1200 & 6.9502 & 6.9523 & 6.9640 & -1.17 \\
1300 & 7.9925 & 7.9951 & 8.0100 & -1.38 \\
1400 & 9.1041 & 9.1072 & 9.1250 & -1.56 \\
1500 & 10.2775 & 10.2812 & 10.2990 & -1.48 \\
1600 & 11.5065 & 11.5108 & 11.5300 & -1.52 \\
\hline \multicolumn{5}{|c|}{ Gas filled lamps } \\
\hline 1600 & 13.8732 & 13.8772 & 13.9110 & -2.91 \\
1700 & 15.0676 & 15.0723 & 15.1150 & -3.46 \\
1800 & 16.3400 & 16.3455 & 16.3920 & -3.57 \\
1900 & 17.6745 & 17.6808 & 17.7360 & -4.05 \\
2000 & 19.0684 & 19.0756 & 19.1400 & -4.52 \\
2100 & 20.5226 & 20.5307 & 20.6030 & -4.88 \\
2200 & 22.0341 & 22.0433 & 22.1250 & -5.30 \\
\hline
\end{tabular}

It can be seen from Fig.(4) that, for the two lamps of NIS, the differences between NIS and NIM ranged from $0.34{ }^{\circ} \mathrm{C}$ at $800{ }^{\circ} \mathrm{C}$ to $-1.52{ }^{\circ} \mathrm{C}$ at $1600{ }^{\circ} \mathrm{C}$ for evacuated lamps, and from $-5.30{ }^{\circ} \mathrm{C}$ to $-2.91{ }^{\circ} \mathrm{C}$ at 1600 to -5.30 at $2200 \mathrm{t}{ }^{\circ} \mathrm{C}$ for the gas filled ones. Nevertheless one possible cause of the differences is due to the difference in the widths of the lamp filaments and it was noticed that the NIS lamps were always higher in radiance temperature than NIM lamps which drives to recommend that the comparison should be repeated at NIS. 
This intercomparison will assist to establish the degree of equivalence of national standards maintained at NIS and NIM. In addition, it provides the mutual recognition of calibration and measurement between the two national laboratories.

\section{References:}

1. H. Preston-Thomas, Metrologia, 27, 3, (1990).

2. H.C. McEvoy, K.M. Raven, A.I. Pokhodoun, M.S. Matveyev, TEMPMEKO'96, 272, (1996).

3. R.D. Lee, H.J. Kostkowski, P.R. Chandler, T.P. Jones, J. Tapping, H. Kunz, Instrument Society of America, 4, 377, (1972).

4. K. Raven, NPL Report CBTM S8, March (1998).

5. D. P. deWoiH and G. Nutter., "Theory and Practice of Radiation thermometry", John wiley and Sons Inc., New York, Ch. 13, (1988)

6. L.N., Latyev, V. Ya. Chekhovskoi, and E.N. Shestakov., High temperatureHigh Pressure, 14, 679, (1972). 